REYISTA

dete

CEPA

NUMERO 62

AGOSTO 1997

SANTIAGO DE CHILE

OSCAR ALTIMIR

Direcsor

EUGENIO LAHERA

Secretario Técnico

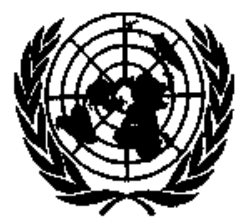

NACIONES UNIDAS 
Estado, comunidad y sociedad en el desarrollo social

Fermando Henrique Cardoso

Un balance de las reformas estructurales neoliberales en América Latina

Joseph Ramos

Deuda y sostenibilidad ffscal: ise repite la historia?

Guillermo E. Perry

Reformas petroleras: las opciones en juego

Fernando Sánchez Albavera

Las organizaciones indígenas: actores emergentes en

América Latina

Rodolfo Stavenhagen

El empleo rural no agropecuario en el Istmo Centroamericano

Jürgen Weller

Marginalidad e integración social en Uruguay

Rubén Kaztman

La política comercial en el marco de la Organización Mundial

de Comercio

Diana Tussie

Comercio y medio ambiente: ¿luz verde o luz roja?

Helga Hoffmann

Anclas nominales y escenarios de coordinación macroeconómica en el MERCoSUR

Gonzalo Rodríguez Prada

Políticas de promoción de exportaciones en Centroamérica

Larry Willmore

Publicaciones recientes de la CEPAL 


\section{Comercio y medio ambiente: ¿luz verde o luz roja?}

\section{Helga Hoffmann}

Directora de la División de Medio Ambiense y Desarrollo, CEPAL
Un aspecto de la globalización que los países de América Latina y el Caribe tendrán que afrontar es el incremento de las restricciones al comercio basado en razones ambientales. No se puede atribuir a la casualidad que la primera diferencia dirimida por el nuevo Organo de Apelación de la Organización Mundial del Comercio (OMC), que empezó a funcionar en febrero de 1996, fuese una controversia ambiental y que implicase a los Estados Unidos y a países de América Latina. Dos tendencias - por un lado economías más abiertas y rápido crecimiento del comercio internacional, y por otro el hecho de que la comunidad internacional reconozca en mayor medida las "responsabilidades ambientales," to que se expresa en djversos acuerdos multilaterales ambientales- han puesto sobre el tapete dos interrogantes: ¿Son las restricciones comerciales un instrumento eficaz para poner en práctica las políticas ambientales? ${ }_{i}$ En qué medida se puede decir que las restricciones ambientales están modificando el comercio y la competitividad a nivel internacional? Quienes formulan las políticas responden de hecho a estas preguntas de manera contradictoria, al tiempo que sólo ahora comienzan a realizarse estudios empíricos para evaluar el efecto de las medidas comerciales relacionadas con el medio ambiente y las medidas ambientales vinculadas con el comercio. 
I

\section{El objetivo y el instrumento: ¿comercio o medio ambiente?}

La interacción de las políticas ambientales y el comercio internacional ha traído a colación dos interrogantes que los responsables de formular las políticas han debido enfrentar y ante las cuales han tomado decisiones, sin tener la posibilidad de esperar la respuesta que podrían proporcionar los académicos y sus investigaciones. Una interrogante comienza con el medio ambiente, la otra con el comercio:

i) ¿Se pueden alcanzar los objetivos ambientales por medio de reglas aplicadas al comercio internacional 0 , dicho de otra manera, son las reglas del comer. cio un instrumento adecuado para alcanzar los objetivos ambientales?

ii) ¿Se están modificando signiticativamente los flujos comerciales internacionales y la competitividad debido al aumento de las restricciones ambientales de variadlo origen?

Quienes contestan a la primera pregunta con un rotunito sí esperan en general propagar y magnificar "el buen cuidado del medio ambiente" a través del comercio internacional, "verdeciendo" a la larga este comercio. Y quienes contestan positivamente a la segunda prigunta ven un incremento de las restricciones al comercio internacional, que proceden de diversas fuen. tes, entre ellas la conciencia ambiental de los consumidores en ciertos países, las cláusulas comerciales en los acuerdos multiłaterales ambientates (AMA), las campañas impulsadas por las grandes organizaciones internacionales de índole no gubernamental, los intereses de las industrias que han logrado una ventaja competitiva en "bienes ambientales" o los intentos de otras indusirias o actividades por aprovechar los argumentos ambientales con fines proteccionistas. Para resumir, se trata de luces rojas en el comercio internacional, impuestas sobre bases ambientales reales o supuestas.

\footnotetext{
1... Este texto se basa en las notas preparadas para una presentación en el sciminario cítulado "Las restricciones ambientales y los desafíos para el comercio internacional y el desarrollo" (Universidad de Chile. Fiacultad de Economía y Administración y Facultad de Ciencias, Dspartamento de Ciencias Ecológieas, 29 a 30 de abril de 1996). Sus principales postulados se presentaron en el "Diálogo entre la Unión Europea y el Grupo de Río sobre el desarrollo sostenible," organizado por el Instituto para las Relaciones entre Europa y América Latina (IRELA) y realizado en La Haya el 27 y 28 de febrero de 1997.
}

Desde este último punto de vista, la preocupación ya no es si el comercio constituye un instrumento útil de política ambiental, sino cómo competir, dado el aumento de las exigencias ambientales en el comercio internacional.

Estas preguntas no son simétricas. Aun si se pudieran obtener suficientes pruebas de que las probibiciones y restricciones comerciales no tienen relación alguna con sus objetivos ambientales expresados -como parece ser el caso, entre otros, de la relación entre la matanza de elefantes y la prohibición del tráfico de marfil ${ }^{1-}$ las restricciones por razones ambientales podrían estar de hecho influyendo en la competitividad y los flujos comerciales. Ambas son, en principio, pregunlas empiricas. Varias prohibiciones y restricciones comerciales se han establecido con propósitos ambientales y debería ser posible examinar si se logró en cada caso una finalidad ambiental específica (proteger los delfines o las tortugas de mar por medio de la prohibición de importar atunes o camarones, reducir la contaminación del aire recurriendo a la prohibición de importar una mezcla determinada de gasolina) y si ese resultado se debió al uso del instrumento comercial y no a otras causas. Que se haya alcanzado un objetivo ambiental especílico y expresado, sin embargo, no significa aún que ese resultado sea el mejor para una prudencia ambiental de conjunto, puesto que, en comparación, la reducción de las importaciones y un menor desarrollo podrían muy bien haber tenido, al final, un mayor impacto negativo en el medio ambiente. Por otra parte, es posible al menos evaluar los efectos que pueden tener medidas ambientales específicas en los flujos comerciales (en qué medida se redujeron las exportaciones de atún, o de gasolina, o de marfil del país $x \circ y)$ y, a la vez, estimar las consecuencias de la disminución del comercio en el empleo y el desarrollo global del país. De ese modo, cuando el país adopta una medida comercial para alcanzar un objetivo ambiental interno, el beneficio ambiental en un extremo puede compararse con la reducción de las exportaciones

\footnotetext{
' Hay ut solprendente número de estudios ambientalistas que mues. tran que la prohibición del tráfico de marfill, si bien redujo el comercio internacional del mismo, no ha contribuido a la conservación de los elefantes (véase Adauns y McShane, 1992).
} 
en el otro, y además con el efecto de la reducción de las exportaciones en el desarrollo del país.

Al formular las preguntas como lo hemos becho es posible evaluar, aunque de manera nuy limitada, los beneficios y perjuicios para las partes en una controversia. $^{2}$ Sin embargo, aun si tuviéramos muchos ejemplos que agregar, con elto no se contestaria la pregunta global en cuanto a si el sistema comercial internacional o el comercio internacional causan deterioro ambiental, en todo el mundo o en países determinados, como siguen sosteniendo unos cuantos ambientalistas. Como ha conclutido David Pearce, destacado economista ambiental, es difícil demostrar que la degradación ambiental que trae consigo el libre comercio se debe realmente al comercio más bien que a algún otro factor, y que la pérdida de bienestar humano que representa es mayor que la pérdida derivada de una disminución del comercio: "como nadie ha calculado aún el valor monetario de las pérdidas ambientales originadas por el comercio internacional, los ambientalistas pueden aducir que ellas son muy cuantiosas en relación con los beneficios obtenidos del comercio. Simplemente no lo sabemos" (Pearce, 1994, p. 35).

Dados los últimos adelantos de la valoración económica de los bienes y perjuicios ambientales, no es exagerado postular que nunca conoceremos las cifras exactas de esta comparación. Siguiendo el mismo razonamiento, no tiene base la imputación de que la actual estrategia de desatrollo de América Latina y el Caribe orientada a la exportación está, en general, dañando el medio ambiente. Aungue podemos tratar de poner en la balanza, por un lado, el valor de $n$ cantidad de tortugas salvadas, y por otro una desaceleración del desarrollo económico del país al que se prohíbe exportar sus camarones, la metodología que puede utilizarse no arrojará cifras exactas, sólo estimaciones. No obstante, vale la pena intentar la valoración, ya que la estimación incluso burda de las ventajas y desventajas comparativas es una base menos riesgosa para formular políticas que la presunción de algunos ambientalistas de que el valor de algo que no se puede medir con precisión es por consiguiente infinito.

Tras examinar numetosos estudios empíricos, Markandya (1994, págs. 10-22) ha planteado estas mismas interrogantes desde un punto de vista algo diferente. Lo que se pregunta es si acaso la liberalización comercial está en pugna con el desarrollo sostenible, y si las políticas conducentes al desarrollo sostenible restringirán el comercio internacional. Su conclusión es que la información disponible con respecto a ambas preguntas es contradictoria, y que de una forma u otra no es significativa. ${ }^{3}$

En todo caso, para formular las políticas no se puede esperar a que se realicen todas las mediciones que habría que llevar a cabo. En cierto sentido, preguntar si el libre comercio es bueno o malo para el medio ambiente tiene tanto significado concreto como preguntar si sería mejor vivir en Marte para proteger el medio ambiente en la Tierra. Las estrategias de desarrollo orientadas hacia la exportación y la integración en la economía mundial se han ido generalizando en e] mundo desarrollado $y$, de forma más radical, en el mundo en desarrolto y es difícil hallar a alguien dispuesto a re:tbrir este debate para detender, basándose en razones ambientales, la ventaja o factibilidad de las estrategias de desartollo hacia adentro.

\section{II}

\section{El contexto internacional}

A las dos preguntas sobre la interacción del comercio y el desarrollo se les da respuesta de hecho en un

\footnotetext{
${ }^{2}$ Nadie ha calculado científicatnente cúnto desarrollo sostenible perdieron México, Venezucla y Colombia a causa del embargo del atún impuesto por los Estados Unidos desde 1991, ni cuánta protección ambiental en favor de los delfines del mundo se obtuvo a cambio. ¿Cuántos de los 25000 empleos en la producción de atún de México se perdieron o se desplazaron bacia actividades dañinas para el medio ambiente? Algunos dicen que la única diferencia entre e] atún del Océano Pacífico asiático e Indico, y el atún de América Latina al que reemplazó, era que aquél se hallaba muy distante de los ojos de los ambientalistas estadounidenses.
}

ámbito en que actúan dos tendencias principales: por una parte, la globalización, que incluso si no es solamente económica, está impulsada por la integración económica mundial y un rápido aumento del comer-

\footnotetext{
${ }^{3}$ En su análisis, Markandya ha recurrido, entre otros, a estudios da casos preparados en el marco del Programa de Irvestigación sobre el Comercio y el Medio Ambiente de UNCTAD/PNud en el que paticipó. Se encuentra entre quienes han descartado cot demasiada facilidad el problema de los costos que han significarlo para los paises en desartollo las nuevas restricciones ambientales en sus mercidos de exportación.
} 
cio internacional; por otra parte, un reconocimiento más jeneral de las "responsabilidades ambientales" de las sociedades y los gobiernos y la admisión de la necesidad de cooperación internacional para hacer frente a tsas responsabilidades. El Programa 21 : Programa de Acción para el Desarrollo Sostenible y la Declaraisión de Rio sobre el Medio Ambiente y el Desarrollo, ${ }^{4}$ así como un número cada vez mayor de acuerdos ambientales multilaterales, constituyen puntos de referencia tan importantes en este sentido como el Acta Final de la Ronda Uruguay aprobada en Marraquech.

Desde 1950 el volumen del comercio mundial de mercincías ha crecido con mayor rapidez que la producción mundial. Es efectivo que durante estos más de cuarenta años ha variado la elasticidad comercio de la producción mundial, que era muy baja entre mediados de los años setenta y mediados de los ochenta, y ha sido inucho más alta en los noventa. En 1995 el ritmo de crecimiento del comercio triplicó casi el de la producción, y a pesar de que se ha estrechado algo la diferenıia, en 1996 casi lo duplicó y se pronostica que aproximadamente lo mismo sucederá en 1997.

Jistas tendencias no pueden atribuirse sólo a las diversas rondas de liberalización comercial del GATT que comenzaron en 1950 y culminaron con la Ronda Urugıay. Se han estudiado otros' factores, como los cambios tecnológicos que han reducido los costos de las ccmunicaciones y el transporte, o el mayor grado de inclustrialización que ha dado lugar a la creación de nuevos productos, a la especialización y a un creciente contercio interindustrial. Sin embargo, lo que se debe destacar es que las políticas gubernamentales han cumplido un papel importante al liberalizar el comercio y establecer un sistema de comercio internacional basado en reglas. Los gobiernos participaron en ocho rondas del GATT, tras las cuales los aranceles medios que los países industriales aplican a las importaciones de manufacturas bajaron a menos de 4\% (CEPAL, 1996, p. 134). Es verdad también que los aranceles de los paises industriales aplicados a productos de mayor interés para los países en desarrollo (en especial los textiles, el cuero y el calzado y los productos agrícolas) son mucho más altos, nuevamente como resultado de las políticas vigentes y de las diferencias en poder de negociación. Aún más importante para el tema que se analiza es que las barreras no arancelarias y las medidas "de zona gris" (incluida la protección ambiental real o supuesta) son hoy parte central de las preocupaciones vinculadas al desarrollo y de las actividades de la OMC (Agosin y Tussie, eds., 1993).

Los países de América Latina y el Caribe no sólo reflejan estas tendencias, sino que además contribuyeron a definirlas. En el período inmediatamente anterior a la Ronda Uruguay los países latinoamericanos se incorporaron al GATT en número creciente y actualmente casi todos son miembros de la oMc. Los gobiernos de América Latina tuvieron una importante participación en la Ronda Uruguay, representados por un pequeño grupo de negociadores muy destacados y competentes. ${ }^{5}$ Una compleja red de acuerdos subregionales de comercio y de integración económica en América Latina y el Caribe y los importantes pasos dados por los países del mercosur en Sudamérica confirman que se ha optado por una firme política en favor de la liberalización comercial y de la integración en la economía mundial.

\section{III}

\section{El comercio como instrumento}

No está demostrada ni mucho menos la eficacia del comercio como instrumento de política ambiental. ${ }^{6} \mathrm{Sin}$ embargo, el comercio internacional se usa cada vez más como instrumento de esta índole, a niveles diferentes, unilateral o multilateralmente, a veces en forma voluntaria — como en el caso de las etiquetas ecoló-

\footnotetext{
${ }^{4}$ Aprobados en la Conferencia de las Naciones Unidas sobre el Medio Ambiente y el Desarrollo, efectuada en Rio de Janeiro en junio de 1992.
}

gicas o algunas de las cláusulas en los acuerdos ambientales multilaterales--, pero otras veces como imposición a países específicos. No fue una casualidad

\footnotetext{
5 Vienen prontamente a la memoria los nombres del fallecido Leopoldo Tettamanti de Argentina; Rubens Ricupero, Georges A. Maciel y Paulo Nogueira Batista -tanbién follecido-- de Brasil: Patricio Leiva de Chile, Felipe Jaramillo de Colombia, Julio Lacarte de Uruguay.

"Vease un completo análisis de los pros y los contras en Low (ed., 1992). Véase también Pearce (1994) y Markandya (1994).
} 
que el primer caso que el nuevo Organo de Apelación de la omc recibió para emitir un informe fuese una con. troversia relativa al "medio ambiente y el comercio." Venezuela, pais al que posteriormente se unió Brasil, había solicitado en 1995 el establecimiento de un panel de la OMC para que examinara su aseveración de que la normativa de los Estados Unidos para la gasolina -siguiendo el reglamento establecido por el organismo estadounidense de protección del medio ambiente (US Environmental Protection Agency) para poner en práctica la ley sobre aire limpio (US Clean Air Act) - aplicaba a la gasolina importada estándares diferentes a los que regían para el producto nacional. El informe que ese panel presentó al Organo de Solución de Diferencias de la omc en febrero de 1996 concluyó que las normas para la gasolina aplicadas por los Estados Unidos no eran consecuentes con las reglas comerciales convenidas internacionalmente; vale decir, que infringían el artículo III del GATT, la disposición sobre "trato nacional" incorporada en los acuerdos de la OMC, según la cual los bienes importados desde páses miembros deben recibir un trato no menos favorable que el otorgado a los bienes originarios del propio país, en todo tipo de legislación.

Además, el panel estimó que las normas de los Estados Unidos para la gasolina que estaban bajo consideración no podían justiticarse en virtud de las excepciones del artículo XX del GATT admitidas en el inciso b): medidas "necesarias para proteger la salud y la vida de las personas y animales y para preservar la vida vegetal"; en el inciso d): medidas para asegurar e] cumplimiento de las leyes o reglamentos que no estén en consonancia con los acuerdos del GATT; $y$ en el inciso g): medidas "relativas a la conservación de los recursos naturales a condición de que tales medidas se apliquen conjuntamente con restricciones a la producsión o al consumo nacionales". Los Estados Unidos recurrieron al Organo de Apelación de la OMC ese mismo febrero de 1996 y casi un año más tarde, en enero de 1997, éste juzgó que de hecho la línea de acción de los Estados Unidos era incompatible con las reglas , Je la omc porque trataba la gasolina de Venezuela y te Brasil en forma menos favorable que la gasolina acional.

Estrictamente hablando, la controversia de la gasolina en la OMC no fue un caso ambiental: no estaban in tela de juicio los objetivos ambientales del país que 'Jiscriminaba las importaciones ni se discutió si dichos objetivos se alcanzaban de manera optima, o incluso si se alcanzaban del todo. La controversia tuvo que ver con el uso del comercio como instrumento para lograr objetivos ambientales. Según el informe del grupo de expertos, los países miembros de la omc son libres de establecer sus propios objetivos ambientales, pero la discriminación comercial no es un instrumento aceptable para tratar de alcanzarlos.

Los acuerdos comerciales regionales y subregionales en América Latina y el Caribe han tendido últimamente a incluir protocolos respecto de los objetivos ambientales que se perseguirán en su comercio o a pesar de su comercio, y algunos hasta han incluido negociaciones orientadas a lograr, si no la armonización, al menos algún grado de compatibilización de las reglas y reglamentos ambientales de las partes con el fin de establecer una base equitativa para una competencia justa entre sus sectores productivos. Lo que importa aquí es identificar las posibles diferencias de intereses y evitar futuras controversias.

Si bien las medidas comerciales unilaterales con fïnes ambientales o incluso las directrices ambientales vinculadas a planes regionales de integración tal vez sean las que más se divulgan, en especial cuando las partes implicadas recurren al mecanismo de solución de controversias de la omc un aspecto muchísimo más importante que merece estudiarse es el efecto actual o potencial de las cláusulas comerciales en un número creciente de acuerdos ambientales multilaterales. Algunas de estas cláusulas, puesto que discriminan entre países, no son compatibles con las reglas de la OMC. Algunas de ellas contemplan la protección del medio ambiente en un país que no es el que impone la medida, adquiriendo de ese modo un alcance extraterritorial que también es incompatible con los principios del libre comercio. Sin embargo, a las medidas comerciales exigidas por los acuerdos mulilaterales ambientales les otorga legitimidad su objetivo expresado de proteger "el legado común de la humanidad", por lo que su impugnación en la omc es menos probable que la de aquellas adoptadas unilateralmente y que invocan leyes nacionales (como la ley de los Estados Unidos relativa a la limpieza del aire, aquella sobre la protección de los mamíferos marinos o las leyes federales del mismo país sobre la protección de las tortugas). Así, se ha formado en los últimos años un importante conjunto de acuerdos multilaterales ambientales, lo que hará más difícil argüir que se justifica una medida unjlateral por no haber reglas multilaterales. 


\section{IV}

\section{El intento de poner en vigor los acuerdos}

\section{multilaterales ambientales}

Existen ya cientos de acuerdos multilaterales ambientales (AMA) de variada cobertura geográfica, a nivel munclial, regional o subregional, que a veces abordan problemas muy específicos. El Programa de las Naciones Unidas para el Medio Ambiente mantiene un registro de dichos acuerdos (PNUMA, 1996). Algunos tienen cláusulas relativas al comercio, que se supone ayudarín a alcanzar sus objetivos ambientales o de desarrollo sostenible, y orros, sin contener cláusulas específicas relativas al comercio, podrían de todos modos afectar a los flujos comerciales. Dado que estos acuerdos multilaterales ambientales son relativamente nuevos, no se cuenta con mucha investigación empírica para evaluar si tienen (o podrían tener) un efecto importante en el comercio internacional. Se examinarán boevemente los más importantes de dichos acuerdos con implicaciones comerciales, para así ilustrar algunas diferencias entre el régimen comercial internacional y los regímenes ambientales. ${ }^{7}$ Este breve análisis nos permitirá hacer una amplia comparación de los regímenes internacionales de comercio y los de medio ambiente.

\section{Reducción de los desechos peligrosos}

En última instancia, el objetivo del Convenio de Basilea sobre el Control de los Movimientos Transfronterizos de los Desechos Peligrosos y su Eliminación (1989) es modificar los patrones de consumo y producción para así reducir al mínimo los desechos peligrosos que se generan en los procesos productivos. Con ese fín, establece una serie de restricciones a su tránsito. El Convenio ha sido ratificado por 105 países, entre ellos la mayoría de los de América Latina y el Caribe.

Iil Convenio permite a las partes contratantes adoptar medidas severas, como prohibir completamente las importaciones y exportaciones de ciertas categorías de de:iechos, incluidas en el anexo I o que están apro-

\footnotetext{
${ }^{7}$ Expresamos nuestros agradecimientos a Carmen Artigas, de la División de Medio Ambjente y Desarrollo de la CEPAL, por la ayuda que prestó en el análisis de los acuerdos multilaterales ambientales.
}

badas como anexo I según las recomendaciones del grupo técnico de trabajo vinculado al Convenio. Además de las categorías de desechos que deben controlarse, hay ciertas categorías permitidas en el comercio internacional bajo determinadas condiciones específicas, que incluyen la obtención del "consentimiento fundamentado previo" del país importador. Se han establecido procedimientos de notificación, y las partes tienen que prohibir las exportaciones al país que no permita la importación específica y también al que no haya dado su consentimiento por escrito. En cuanto a los desechos calificados de peligrosos de conformidad con los procedimientos estipulados por la Convención, se prohíben los desplazamientos entre las partes contratantes y las que no lo son, salvo que haya acuerdos bilaterales y multilaterales que estipulen el mismo grado de protección ambiental que el Convenio.

El hecho más reciente respecto del movimiento transfronterizo de desechos peligrosos es la decisión consensual de las partes contratantes del Convenio de incorporar una enmienda que prohibirá a los países desarrollados transportar desechos peligrosos para su reciclaje en países en desarrollo. Hasta ahora muy pocos países han ratificado la enmienda, que debería entrar en vigencia en enero de 1998. Los gobiernos esperaban una aclaración del grupo técnico de trabajo del Convenio respecto de qué materiales para reciclaje abarcaría la enmienda.

En febrero de 1997 el grupo téenico de trabajo recomendó nuevas listas: de desechos calificados de peligrosos según el artículo I, párrafo 1.a del Convenio - (lista A)-; de desechos no considerados peligrosos de acuerdo con el mismo artículo - (lista B) - (PNUMA, 1997a) y de desechos colocados en una lista $C$, especie de lista de espera de residuos por considerar para su inclusión en la lista A o B (PNUMA, 1997b). Naturalmente, éstas son sólo recomendaciones a la Conferencia de las Partes firmantes del Convenio.

Hay otras incertidumbres. Una se refiere a las reglas de Ja omc relativas al comercio de productos que se han de reciclar. ¿Qué sucede cuando un país se incorpora a la Organización de Cooperación y Desarrollo Económicos (OCDE)? ¿Pierde el derecho a comerciar con sus vecinos fuera de la OCDE y de acceder al 
comercio con países desarrollados, los que, según la enmienda, mantienen su derecho a comerciar entre sí?

El grupo técnico de trabajo del Convenio había recomendado a fines de 1995 excluir de las restricciones ciertas categorías de desechos reciclables, consistentes en metales y compuestos de metales en forma no dispersable, siempre que se hubiesen manipulado conforme a ciertas reglas y no incluyesen desechos comprendidos en el Convenio o componentes que los hiciesen peligrosos. Los desechos reciclables que debían excluirse eran los siguientes: de metales preciosos (excluido el mercurio), de hierro y de acero, de cobre, y de varios otros metales, como níquel, aluminio, zinc, tungsteno, molibdeno y manganeso. Esta recomendación se confirmó en la última reunión del grupo técnico de trabajo, en febrero de 1997.

El debate del grupo técnico ilustra una de las dificultades que tiene que enfrentar el Convenio, vale decir, la tenue línea entre lo que es un producto y lo que es desecho. Otra dificultad general es la de alcanzar un acuerdo sobre qué es "peligroso" para justificar la inclusión en la lista de "desechos peligrosos" (PNUMA, 1997b). El Convenio en su anexo III incluye y define las "características peligrosas" de un material: explosivo, inflamable, propenso a la combustión espontánea, venenoso, infeccioso, conosivo, tóxico, ecotóxico, etc. No obstante, la peligrosidad varía de un material a otro, según la dosis y la reacción correspondiente, el tiempo de exposición y, en el caso de las amalgamas o combinaciones, según la sinergia. Lo más importante es que la peligrosidad depende de la existencia (o inexistencia) de una tecnología adecuada para la manipulación y el reciclaje, y de la capacidad de manejar los desechos peligrosos de manera segura desde el punto de vista ambiental.

Para alentar alguna esperanza de que haya un control eficaz de los movimientos transfronterizos de desechos peligrosos y se evite el transporte ilegal será nezesario también estandarizar las nomenclaturas. La que se usa en relación con el Convenio de Basilea, basada zn la lista de productos peligrosos elaborada por las Naciones Unidas, no coincide con la Clasificación Uniforme para el Comercio Internacional, de las $\mathrm{Na}$ jiones Unidas, ni con la Nomenclatura para la Clasifjcación de Mercancías en los Aranceles de Aduana del Consejo de Cooperación Aduanera, la llamada Nomen- latura Arancelaria de Bruselas que usan las autorida. Jes de comercio. ${ }^{8}$ Su efícaz puesta en práctica se hace

\footnotetext{
"Sirve para ilustrar estas jucertidumbres el caso de unos contenedores de selenio que las autoridades chilenas de salud consideraron
}

aún más compleja por la multiplicidad de instrumentos para la "seguridad química", entre ellos el Código internacional de conducta para la distribución y utilización de plaguicidas de la Organización de Jas Naciones Unidas para la Agricultura y la Alimencación (FAO) o las Directrices de Londres para el intercambio de información acerca de productos químicos objeto de comercio internacional, que intentan prohibir o restringir ciertas sustancias químicas y adoptar procedimientos de consentimiento fundamentado previo para muchas otras. Por último, pero no por eso menos inportante, la falta de una tecnología adecuada y de capacidad técnica, en especial en los países en desarrollo signatarios del Convenio, constituye un impedimento adicional. Los países de América Latína y el Caribe estudian actualmente la creación de una red de centros regionales de capacitación y apoyo técnico con miras a la puesta en práctica a nivel nacional de lo estipulado en el Convenio, y de transferencia de tecnología que propicie una gestión racional de los desechos peligrosos (CEPAL, 1995).

Un problema vinculado a los desechos peligrosos que el Convenio de Basilea intenta resolver es el del tratamiento que debe darse en el comercio internacional a las "mercancías prohibidas en el país." Esta es una categoría más amplia, que abarca en especial los fármacos, bienes de consumo, productos químicos, cosméticos y alimentos cuya venta en el mercado interno no se permite, o para la cual no se ha obtenido un permiso legal, o que tiene un permiso que está vencido. Puede abarcar también maquinaria y equipo o insumos prohibidos en el país exportador por riesgos para la salud o la seguridad. En el Comité de Comercio y Medio Ambiente de la omc continúan los debates sobre esta materia.

\section{Diversidad biológica}

Entre los innumerables acuerdos y mecanismos de consulta relativos a la conservación de la naturaleza, la Convención sobre el comercio internacional de especies amenazadas de fauna y flora silvestre (CTTES) es el más antiguo en cuanto a utilizar explícita y principalmente "medidas ambientales relacionadas con el

\footnotetext{
desechos petigrosos (en virtud det Convenio que Chile ha ratificado), en tanto que la Corte Suprema de Chile, en noviembre de 1994 , declaró que se trataba de un producto comercial que ingresaba al pais ajustado a los reglamentos ("Corporación Minera y Química Cormiquin SA con Servicio de Salud del Ambiente", Rol de ingreso Corte 2552-94)
} 
comercio". La crtes, que cuenta con 134 signatarios, se adoptó en Washington en 1973 y se enmendó en 1979 y en 1983. Prohíbe el comercio de especies amenazadas de extinción y regula el comercio de especies que podrían llegar a estar en peligro. La conferencia de las Partes contratantes de la Convención decide regularmente, por una mayoría de dos tercios, sobre criterios científicos y sobre inclusiones y exclusiones en el apéndice I, la lista de todas las especies amenazadas de extinción y que requieren una "reglamentación particularmente estricta" o prohibiciones de comercio; en el apéndice II, la lista de las especies no amenazadas de extinción ahora pero cuya supervivencia justifica las restricciones comerciales, y en el apéndice III, la lista de otras especies que necesitan la cooperación de las Partes. Para llevar esto a la práctica, los países tienen que imponer restricciones a las importaciones $o$ a las exportaciones, según la ubicación geográfiça de la fauna o flora en cuestión.

El comercio con países que no han firmado la Convención está sujeto en principio a las mismas reglas que las que se aplican a los signatarios. La Unión Europea ya ha aprobado reglas para la aplicación de la cries en Europa que son más rigurosas que las rẹglas de la Convención y ha incorporado especies que ésta tro incluye. Con objetivos relacionados con los de la CITES hay una nueva Convención sobre los animales migratorios, bajo cuyos auspicios se han adoptado alguno: acuerdos regionales, sobre la conservación de los cetáceos de los mares Báltico y del Norte, de los murciélał̧os en Europa, de las aves acuáticas africano-eurasianas. Sin embargo, en estos casos se hace menos hincapié en medidas comerciales.

ha Secretaría de la cites en Ginebra verifica las infracciones e informa a la conferencia de las Partes sobre los problemas de ejecución. La IX conferencia de la:i Partes que se reunió en Fort Lauderdale, Estados Unidos, en 1994 aprobó por unanimidad nuevos criterios para la elaboración de las listas y examinó las infracciones. Ejemplos recientes incluyen el descubrimiento de una caja con un gorila y un chimpancé en el aeropuerto de Kigali (Ruanda), contenedores con esculturas de marfil en Bruselas, la piel de una serpiente en un paquete postal enviado desde Argentina a Dinamarca (cITES, 1994). La protección de la fauna atrae mucha publicidad, y la crTes edita una revista trimestral (CrrES/C\&M International Magazine); pero por la pequeña escala y la índole del comercio que examina, la crTes no se ha situado en el centro de las inquietudes sobre la compatibilidad entre el régimen comercial y el medio ambiente.
A pesar de su carencia de cláusulas comerciales explícitas, la puesta en práctica del Convenio sobre la Diversidad Biológica podría tener una importancia mucho mayor en el comercio internacional. Este Convenio se presentó para su firma en la Conferencia de las Naciones Unidas sobre el Medio Ambiente y el Desarrollo (1992) y actualmente, con la notable excepción de los Estados Unidos, casi todos los países lo firmaron y ratificaron: 163 países a fines de 1996 , entre ellos la mayoría de los de América Latina y el Caribe.

Sus principales objetivos son la conservación y uso sostenible de la diversidad biológica y la distribución equitativa de sus beneficios o, según se formula en su artículo I, "... la conservación de la diversidad biológica, la utilización sostenible de sus componentes y la participación justa y equitativa en los beneficios que se deriven de la utilización de los recursos genéticos, mediante, entre otras cosas, un acceso adecuado a esos recursos y una transferencia apropiada de las tecnologías pertinentes, teniendo en cuenta todos los derechos sobre esos recursos y a esas tecnologías, así como mediante una financiación apropiada". Con ese fin, los países se han puesto de acuerdo respecto de una lista completa de medidas para la conservación in situ y ex situ, para la identificación y vigilancia de la diversidad biológica, y para la integración de la conservación y uso de la diversidad biológica en las políticas y programas sectoriales pertinentes.

El Convenio considera que la conservación de la diversidad biológica es "un interés común de la humanidad", pero reafirma la soberanía nacional de los Estados respecto de su propia biodiversidad, que se da a tres niveles: genes, especies y ecosistemas.

De conformidad con el Convenio, cada parte promete facilitar el acceso a sus recursos genéticos, "según mutuo acuerdo" y con sujeción a un consentimiento fundamentado previo (los famosos procedimientos para consentimiento de varios otros acuerdos multilaterales ambientales ). Un punto no resuelto en el debate es cómo compensar a los países en desarrollo por el uso mundial de la diversidad biológica originada en su territorio. El tema es importante para América Latina y el Caribe, región relativamente rica en diversidad biológica, cuyo esquema de desarrollo conlleva la exportación intensiva de recursos naturales. Lo que está en juego aquí es el uso internacional y la asignación de precios a la diversidad biológica, ya que se sabe que está irregularmente distribuida en todo el mundo y que se concentra, por el momento, en los países en desarrollo. 
Se ha sostenido que la diversidad biologica podría convertirse en la nueva ventaja competitiva de estos países (Feinsilver, 1996), lo que podría depender de la posibilidad de negociar el acceso a los recursos de la diversidad biológica a cambio del acceso a tecnología, en especial a la biotecnología, para su uso sostenible. Desde que dieron su conformidad al Convenio sobre la Diversidad Biológica, muchos países en desarrollo han aprobado reglamentos para el acceso a los recursos biológicos y para su exportación, con el propósito no sólo de controlarlos sino también de captar los beneficios económicos que deriven de ellos.

La experiencia práctica más divulgada es el acuerdo de 1991 entre Merck y el Instituto Nacional de la Biodiversidad de Costa Rica (INBio), para la prospección de la diversidad biológica en Costa Rica y la investigación de la posibilidad de obtener y explotar comercialmente fármacos basados en la muestra proporcionada por el INBio. Este organismo recibió inicialmente un millón de dólares para la identificación, recolección y extracción de varias plantas e insectos. Las patentes de los inventos derivados de estas muestras pertenecerían a Merck, pero el INBio tendría derechos sobre los productos farmacéuticos o agroquímicos producidos a base de la muestra proporcionada. ${ }^{9}$

Otra propuesta que se ha planteado es el estudio de un mecanismo internacional aceptable, según el cual los países con diversidad biológica importante para el nundo puedan cobrar por los "servicios ambientales" que su biodiversidad proporciona al mundo (Naciones Unidas, 1997). Los recursos obtenidos de esa forma jodrían utilizarse para una gestión sostenible de esa tiversidad biológica.

Otras cuestiones no resueltas relacionadas con el Convenio son las siguientes:

i) los derechos de los agricultores, es decir, los derechos que se derivan de los aportes pasados, presenles y futuros de los agricultores en cuanto a conservar, mejorar y hacer accesibles los recursos titogenéticos, y que debieran garantizarles la participación en los beneficios resultantes de la fitogenética y de otros métodos científicos aplicados a esos recursos;

ii) el acceso a colecciones ex situ que no fueron al resultado de decisiones del Convenio;

iii) la manera de enfrentar la posibilidad de que unevas variedades uniformes de plantas desplacen la

\footnotetext{
${ }^{5}$ En Feinsilver (1996), se hace un excelente análisis de la experien(ia de Costa Rica y de otros casos de prospección de la diversidad tiologica y su uso comercial y científico, asi como de sus posibilidades para los parses en desarrollo.
}

diversidad biológica que tienen las variedades tradicionales, $y$

iv) el efecto de productos agroquímicos que han permitido ampliar la producción agrícola.

Las cuestiones relativas a la propiedad intelectual ocupan un lugar preponderante en las disposiciones sobre la transferencia de tecnología, en especial la biotecnología, y abarcan no sólo la tecnología para la conservación y el uso sostenible de la diversidad biológica, sino también la que deriva del uso de los recursos genéticos cuyo accceso es objeto del Convenio. La compatibilidad con las reglas de propiedad intelectual de la Organización Mundial de la Propiedad Intelectual (OMPI) y de la OMC es una materia que merece examinarse. En principio, el acuerdo de la omC referente a los derechos de propiedad intelectual relacionados con el comercio no impide a los gobiernos limitar la investígación sobre tecnologías y el uso de las mismas con el propósito de proteger el medio ambiente. Una patente inscrita garantiza que su propietario puede evitar su uso por terceros (salvo excepciones), pero no garantiza al propietario el derecho a explotar la patente si se determina que es perjudicial o peligrosa. No obstante, a las consideraciones que tienen que ver con la seguridad biológica se suman las vinculadas a la apropiación de los beneficios económicos de la explotación comercial de inventos logrados gracias a la diversidad biologica de países que no cuentan con capacidad científica y de investigación para explotar independientemente su propia biodiversidad.

\section{La reducción de las emisiones mundiales de gases de efecto invernadero}

El más reciente de los acuerdos multilaterales ambientales con implicaciones comerciales es la Convención Marco de las Naciones Unidas sobre el Cambio Climático, de 1994. También se presentó para su firma en la Conferencia de las Naciones Unidas sobre Medio Ambiente y Desarrollo en 1992, y entró en vigor en marzo de 1994. Contaba con 163 signatarios a fines de 1996. El objetivo general del acuerdo es proteger el sistema climático de los cambios inducidos por el hombre, y con esa finalidad los gobiernos se han comprometido a una serie de medidas para obtener "Ia estabilización de las concentraciones de gases de efecto invernadero en la atmósfera a un nivel que impida interferencias antropógenas peligrosas en el sistema climático. Ese nivel debería lograrse en un plazo suficiente para permitir que los ecosistemas se adapten naturalmente al cambio climático, asegurar que la pro- 
ducción de alimentos no se vea amenazada y permitir que el desarrollo económico prosiga de manera sostenible" (artículo 2). Además, "Las medidas adoptadas para combatir el cambio climático, incluidas las unilaterales, no deberían constituir un medio de discriminación arbitraria o injustificable ni una restricción encubierta al comercio internacional" (artículo 3).

Al igual que en otras convenciones ambientales importantes, junto a la Convención Marco se encuentra un organo subsidiario de asesoramiento científico y tecnológico para vincular la información y evaluaciones científicas proporcionadas por los organismos internacionales competentes con las necesidades orientada: a las políticas de la conferencia de las Partes. La relación entre el órgano subsidiario y el Grupo Intergubernamental de Expertos sobre Cambios Climáticos (IPCC) no es clara, pero el IPCC confirmó, en su últinıa evaluación, que ha ocurrido un recalentamiento de la Tierra ocasionado por las actividades humanas. ${ }^{10}$

La Convención Marco, así como la primera conferencia de las Partes realizada en Berlín en 1995, insistió en "responsabilidades comunes pero diferenciadas", "capacidades respectivas" y "equidad", por razones obvias, puesto que las principales emisiones que se deben estabilizar son las de díxido de carbono (CO,) y sus fuentes principales son los sectores de transporte y de energía. La mayoría de los países industrializados, como los de la Unión Europea, Japón y los Estados Unidos (los países del anexo I en el habla de esta Convención) se habían comprometido a "volver individual o conjuntamente a los niveles de 1990 de esas emisiones antropógenas de dióxido de carbo. no y otros gases de efecto invernadero" en el año 2000 (artículo 4). No obstante, las metas no son vinculantes y al realizarse la primera conferencia de las Partes en Berlín en 1995 y nuevamente con ocasión de la segunda en Ginebra en 1996, los marcos cronológicos se alargaron aún más hacia el futuro.

Potencialmente, la aplicación de medidas severas contra las emisiones de dióxido de carbono podrían afeciar a la competitividad y al comercio a través de los sectores de la energía y el transporte, ya que ellos proporcionan insumos a todos los procesos de producción. Sin embargo, a pesar de contar con defensores muy respetables (Cline, 1992) y el respaldo en febre-

\footnotetext{
"'Sin embargo, durante la última conferencia de las Partes, en 1996, algunas cuestionaron las conclusiones, y tampoco es evidente que haya un consenso entre los científicos, como queda de manifiesto en Enısley, ed., 1996.
}

ro de 1997 de 2000 economistas estadounidenses entre los que se hallaban seis premios Nobel, la idea de un impuesto internacional sobre la emisión de $\mathrm{CO}_{2} \mathrm{O}$ sobre el uso de combustibles, que podría afectar al comercio de combustibles, no tiene por el momento suficiente apoyo político para convertirse en una propuesta viable.

El instrumento internacional que se está ensayando al poner en práctica la Convención Marco es el de "aplicación conjunta" o de "actividades aplicadas conjuntamente", hoy en una etapa experimental, que podría crear condiciones propicias para adoptar certificados de emisión comercializables en el ámbito internacional. Lo que originó la "aplicación conjunta" fue la idea de que un país (rama de la industria, compañía) pođría cumplir sus obligaciones de reducción de emisiones mediante una combinación de reducciones internas y compensaciones externas." "Esto debería alentar a los países industriales a patrocinar proyectos y estimular la transferencia hacia los países en desarrollo de tecnologías de bajas emisiones.

Están en vías de planificación o de ejecución en los países en desarrollo varios proyectos de "aplicación conjunta" en materia de eficiencia en el uso de la energía, el cambio de combustible, la forestación y la repoblación forestal, especialmente en América Latina y el Caribe (Belice, Costa Rica, Ecuador, Honduras, México, Nicaragua); pero desde el punto de vista de la reducción deseada de las emisiones de gases que causan el efecto invernadero, esos proyectos son insignificantes (Zollinger y Dower, 1996). En esta etapa experimental, no se ha puesto en práctica sistema alguno para otorgar "créditos por reducción de emisiones" a los países patrocinantes. Antes que se puedan dar y comercializar los "créditos", habría que ponerse de acuerdo en un límite, de alcance mundial y verificable, a las emisiones totales, así como en una distribución de las cuotas de emisiones en virtud de la cual las personas y los países más ricos tuviesen que hacerles sitio (ecológico) a los más pobres.

\section{Eliminación de sustancias que agotan la capa de ozono}

El Convenio de Viena sobre la protección de la capa de ozono de 1985, su Protocolo de Montreal relativo a las sustancias agotadoras de la capa de ozono de 1987

\footnotetext{
"Compensaciones significa aquí créditos por reducción de las emisiones, los que una vez oficialmente certificados, podrían comercializarse internacionalmente (Simonis, 1996, p. 100).
} 
y los posteriores ajustes a éste tienen como objetivo proteger la capa de ozono y en último término eliminar la producción y el consumo de las sustancias que la agotan, comenzando con "medidas preventivas para controlar equitativamente las emisiones mundiales totales" de esas sustancias, ${ }^{12}$ en especial los clorofluorocarbonos (CFC). El Protocolo de Montreal, que tiene actualmente 160 signatarios, ${ }^{13}$ concibe una reducción gradual de la producción de sustancias agotadoras de la capa de ozono controladas (CFC y varias otras substancias); para los países en desarrollo que estén bajo cierto nivel de producción de esas sustancias el período de reducción gradual es de 10 años (que se cumpliría en 1999). El Protocolo tiene un grupo asesor de expertos técnicos y económicos y un pequeño fondo fiduciario multilateral, el que ha recibido alrededor de 500 millones de dólares desde su creación en 1991 y que otorga créditos en condiciones muy favorables para ayudar a los países en desarrollo a sulragar los costos relacionados con la aplicación del Protocolo.

Respecto de las sustancias controladas, las partes en el Protocolo han acordado prohibir sus importaciones desde y hacia los Estados que no sean partes en él. La prohibición está vigente para los CFC y halones desde comienzos del decenio de 1990 , y pese a que no es compatible con el principio de no discriminación en el comercio internacional, no se la ha objetado en la OMC.
Los países industrializados se encuentran bien avanzados en el proceso de eliuninación gradual de los CFC y halones, y también muestran progresos con respecto a otras sustancias que agotan la capa de ozono. El Protocolo de Montreal suponía que los países industrializados ya habrían prohibido los halones en 1994, así como los CFC y el tetracloruro de carbono en 1996. Dichos países eliminarán gradualmente el bromuro de metilo a más tardar en el año 2010 y los países en desarrollo han acordado congelarlo a más tardar el año 2002 a los niveles medios de 1995-1998, decisión difícil puesto que con esta sustancia fumigante se combaten las plagas de muchos cultivos de exportación de estos países. Se permitirán excepciones para ciertas aplicaciones relacionadas con el comercio (como inspección previa al embarque y cuarentena) y para usos agrícolas de suma importancia.

Los Estados Unidos dejaron de producir CFC para el consumo interno, salvo para unos cuantos usos esencjales, pero al parecer esto fue el resultado fundamentalmente de medidas internas (Cook, 1966). También ha habido avances en los países en desarrollo, que deberán eliminar gradualmente los CFC antes del año 2010 . No se ha evaluado si las prohibiciones comerciales desempeñaron un papel importante en el cumplimiento de las exigencias de eliminación gradual establecidas por el Protocolo de Montreal.

\section{Comparación entre los regímenes ambientales y el régimen comercial en el ámbito mundial}

Los regímenes ambientales a nivel mundial y el régimen comercial internacional son diferentes: tienen objetivos y estructuras diferentes, funcionan según prin-

\footnotetext{
12 Preámbulo del Protocolo de Montreal. Además de los clorofluorocarbonos (CFC) y halones, el Protocolo y sus enumiendas abarcan otras substancias controladas como el tetracloruro de carbono, el cloroformo de metilo y el bromuro de metilo. Los hidroclorofluorocarbonos se clasifican como sustancias de transiiión, a las cuales se aplican plazos menos rígidos. Se pueden agregar productos a medida que se establece su posibilidad de agotar la zapa de ozono.

${ }^{13}$ Se aprobaron enjniendas al Protocolo de Montreal en posteriores tonferencias de las Partes. A fines de 1996, la enmienda de LonJres de 1990 había sido ratificada por II l países y la etmmenda de Copenhague de 1992 , por 62 países.
}

cipios diferentes, con instituciones e instrumentos diferentes. El uno no se puede convertir en el otro.

Los regímenes ambientales son mucho más complicados que el régimen comercial internacional. Este último se basa en una teoría coherente que muestra que el comercio mejora el bienestar tanto de los exportadores como de los importadores. En cambio los regímenes ambientales, en especial los principales acuerdos multilaterales ambientales ratificados en relación con el Programa 21, promueven el "desarrollo sostenible", concepto vago que cuenta con un amplio apoyo político pero para el cual no hay una definición rigurosa dentro de un marco teórico determinado. Las múltiples definiciones que se usan son esencialmente variantes de la fórmula original de la Comisión Brund- 
tland: "El desarrollo duradero es el desarrollo que satisface las necesidades de la generación presente sin comprometer la capacidad de las generaciones futuras para satisfacer sus propias necesidades" (CMMAD, 1987, p. 59).

Hay un consenso más o menos general respecto del nexo entre actividad económica y ambiente natural, y respecto de los componentes inseparables del desarrollo: el crecimiento económico o aumento selectivo de la producción, el desarrollo social (centrado, en los países en desarrollo, en la lucha contra la pobreza y en la equidad), y la sostenibilidad ambiental que exige la conservación y una justa distribución del uso de los recursos ecológicos. Mucho se ha dicho sobre estas interrelaciones, que ya habían sido puestas de relieve por Gro Harlem Brundtland en marzo de 1987, en su "Prefacio del Presidente" al informe de la comisión independiente establecida por el Secretario General de las Naciones Unidas en 1983 (CMMAD, 1987, págs. ix-xv). Las organizaciones internacionales, en particular las Naciones Unidas, necesariamente suscriben el principio del "desarrollo sostenible" en el enfoque que surge de la Conferencia de las Naciones Unidas sobre el Medio Ambiente y el Desarrollo de 1992. Sin embargo, no hay que investigar demasiado para reconocer las dificultades del concepto.

¿La sostenibilidad de qué? ${ }^{14}{ }_{i}$ Primero el ser humano o primero el ecosistema? Entre el planteamiento antropocéntrico y el ecocéntrico, hay toda una gama de posiciones intermedias frente a los problemas tanto a rivel mundial como a nivel local. Luego el optimismo o pesimismo científico se manifiestan en los supueslos sobre la posibilidad de sustitución entre los propios recursos naturales y entre el capital creado por el hombre y el capital natural (ambos dependientes de los avances tecnológicos).

¿La sostenibilidad dónde? Los diferentes problemas ambientales tienen diferente alcance geográfico. El daño rara vez ocurre exactamente donde se originó. Además, la geografía también es importante en cuanto al efecto de las políticas. ${ }^{15}$ ¿Cómo hacer frente al problema de los recursos comunes y compartidos?

\footnotetext{
${ }^{14}$ EJ profesor Opschoor, Rector del Instituto de Estudios Sociales de La Haya, y al que no se puede acusar de no ser un experto en medio ambiente, dictó una magnífica conferencia sobre la falta de claridad del concepto para responder a esa pregunta y a las de dónde, cuándo y con cuánto riesgo (Opschoor, 1996). He recurrido a sus observaciones.

is Destle el punto de vista de un modelo sistemático de extracción y uso de los recursos naturales, suponiendo dos países, uno de origen y otro extranjero, y adoptando un concepto de "sostenibilidad
}

¿Cómo distribuir geográficamente esas famosas "responsabilidades comunes pero diferenciadas" del Principio 7 de la Declaración de Rio? ¿Cómo establecer el nivel global de protección o uso del medio ambiente ecologico y cómo dividir las cuotas de acceso y uso?

¿La sostenibilidad cuándo? Mejorar el bienestar de las generaciones presentes sin arriesgar la capacidad de las generaciones futuras para mejorar su bienestar es la defínición del consenso amplio. ¿Cuánto riesgo, sin embargo, es aceptable en la evaluación de la equidad entre las generaciones? Además, persiste siempre la cuestión de la incertidumbre científica y sostenibilidad. Ante la incertidumbre, ¿qué nivel de riesgo es aceptable? ${ }_{i}$ Cuán peligroso es, por ejemplo, que se estén postergando los plazos para reducir las emisiones de gases que causan el efecto invernadero? ¿Cuáles son exactamente los plazos que deben establecerse para cada programa de acción con el que se trata de resolver los problemas ambientales? Por último, y vinculado con todas las preguntas anteriores, ¿cómo medir el "capital ambiental" o los "beneficios ecológicos" y la sostenibilidad o la falta de ella?

Los viejos problemas comerciales y la medición de los flujos de comercio parecen, en comparación, sencillos. El sistema de reglas para el comercio internacional se ha estructurado durante más de 50 años, basado en los principios de no discriminación, reciprocidad y trato nacional. Entretanto, los principios y la mayor parte de las reglas actuales para la ordenación ambiental internacional y el "desarrollo sostenible" son relativamente recientes y aún no tienen fuerza legal. En tanto que las negociaciones de la Ronda Uruguay han fortalecido el mecanismo de solución de controversias en el comercio internacional, no hay un mecanismo unificado para la solución de controversias relacionadas con el daño ambiental y su atenuación.

Es cierto que a lo largo de los años se amplió el alcance del régimen comercial internacional, que además del comercio de mercancías pasó a incluir servicios, medidas de inversión relacionadas con el comercio y derechos de propiedad intelectual relacionados

débil", es decir, dejando margen para la sustitución entre el capital creado por el hombre y el natural, Klepper y Stuehler (1996) han demostrado que la adopción unilateral de "reglas de sostenibilidad" en el país de origen se traduce en mayor insostenibilidad en otros paises. Debe señalarse que las "reglas de sostenibilidad" usadas en el modelo son "reglas de extracción de los recursos agotables" y que en el inodelo se postulan mercados perfectamente competitivos (y por lo tanto no hay perturbación del comercio). A menos que la insostenibilidad en el extranjero no sea un problema, los resultados del modelo implican una condena de las reglas unilaterales en un país para la protección del medio aubiente. 
con el comercio. Aun así, en comparación, los regímenes ambientales, en especial el Programa 21, podrían en principio abarcarlo todo. Los problemas ambientales son innumerables, incluso si se tienen en cuenta sólo aquellos con implicaciones transnacionales: la contaminación transfronteriza del aire y el agua, las normas sanitarias para el comercio de alimentos, los productos químicos tóxicos, los desechos peligrosos, el agotamiento de la capa de ozono, la contaminación del mar derivada de fuentes de tierra firme, la contaminación proveniente de los barcos, la pesca excesiva, las especies en peligro de extinción, la pérdida de la diversidad biológica, la erosión del suelo, la desertificación, la deforestación, el cambio ćlimático, etc. Cada uno de ellos involucra a diversas instituciones nacionales y diversos niveles de acción gubernamental (desde comunales a internacionales), origina un buen número de duplicaciones y conflictos jurisdiccionales y mueve a diversas organizaciones internacionales. Además, cada uno se vincula con diferentes actividades e intereses económicos, y no afecta de la misma manera a todos los grupos y estratos socioeconómicos.

A esta cantidad de problemas corresponde un número aún mayor de instrumentos que se usan pata lograr resultados: acuerdos voluntarios entre el gobierno y las industrias; disposiciones que regulan las normas de emisión, de productos, de procesos; exigencias de evaluación, fiscalización e informes ambientales; reglas para ensayos, embalaje y etiquetado.

Añádanse a éstos los incipientes "instrumentos əconómicos," que ahora están bastante en boga: impuestos de diversos tipos (destinados o no a gastos aspecíficos ambientales), cargos por contaminación, Jlanes de reembolso de depósitos, "protocolos verdes" jara créditos (que vinculan el crédito con las evaluaiones de los efectos ambientales), subsidios (que se Jtorgan o se eliminan), tasas y gravámenes a los deseshos, etiquetas ecológicas en la comercialización, insrumentos basados en la responsabilidad civil y permi:sos comerciables (relacionados con el uso de ciertos recursos o con la emisión de sustancias específicas).

Luego está el papel de las ciencias naturales. El régimen comercial se ocupa solamente de las relaciones económicas y no le conciernen los fenómenos naturales. Los acuerdos multilaterales ambientales son radicalmente diferentes. Incluso si intentan abordar las relaciones sociales y económicas, es solamente si ellas ijenen como resultado cambios en la naturaleza, diagnosticados como daño. Aunque el impacto de la degradación ambiental puede a veces percibirse sin preparación científica, han sido los expertos en ciencias naturales, incluidos los especialistas en salud, quienes han identificado la mayoría de los fenómenos ambientales y quienes los siguen de cerca.

No es por azar que casi todos los acuerdos multilaterales ambientales cuenten con un órgano de asesoramiento científico, para la evaluación de los procesos químicos, físicos o meteorológicos, para clasificar los productos tóxicos y peligrosos, etc. No obstante, algunas veces las Partes en las Convenciones o Convenios pasan por alto o incluso cuestionan el asesoramiento científico de dichos órganos.

Para hacer frente a la incertidumbre científica, los regímenes ambientales internacionales han adoptado el principio de precaución, según el cual "Cuando haya peligro de daño grave o irreversible, la falta de certeza científica absoluta no deberá utilizarse como razón para postergar la adopción de medidas eficaces en función de los costos para impedir la degradación del medio ambiente" (como expresa el Principio 15 de la Declaración de Rio sobre el Medio Ambiente y el Desarrollo, 1992). La referencia a "medidas eficaces en función de los costos" es un recordatorio de que el principio de precaución por sí solo es insuficiente para guiar la toma de decisiones, y que no se puede dar por sentado que el valor de evitar el daño ambiental es infinito. Como el daño ambiental y las políticas para evitarlo generalmente dejan ganadores y perdedores, no será fácil coincidir respecto a cuáles son las medidas eficaces en función de los costos, tanto a nivel local como internacional,

Esta variedad de tendencias se han incorporado necesariamente en el sistema de negociaciones ambientales multilaterales. Así por ahora, los mecanismos de puesta en vigor y de solución de controversias en los acuerdos ambientales multilaterales son muy deficientes. Se hace hincapié en los compromisos voluntarios y el intercambio de información, y también en los procedimientos reactivos de las políticas internas, como la aprobación de "estrategias nacionales" en respuesta a acuerdos multilaterales ambientales. De hecho, las medidas comerciales son casi el único instrumento coercitivo en estos acuerdos. ${ }^{16}$

Con el creciente número y variedad de los acuerdos multilaterales ambientales en vigor, y su rápida evolución mediante nuevos protocolos y enmiendas, se está creando una red de regímenes ambientales internacionales cada vez más compleja. Al no haber proce-

\footnotetext{
If Von Moltke (1996) hace una comparación muy esclarecedora entre los regímenes ambientales y comerciales en el plano internacional.
} 
dimientos e incentivos internacionales para su cumplimiento, muchos países -en especial aquellos en desarrollo y entre ellos los de América Latina y el Caribe- no han establecido aún las instituciones y la capacidad técnica necesarias para poner en vigor los acuerdos que han suscrito y fiscalizar su cumplimiento.

Los problemas ambientales que los acuerdos de esta índole tratan de abordar están vinculados entre sí; es cada vez más importante entonces compatibilizar tales acuerdos y coordinar las instituciones internacionales y nacionales que se ocupan de llevarlos a la práctica. El PNUMA, que proporciona apoyo administrativo a diversas secretarías de los acuerdos multilaterales ambientales —en tanto que éstos tienden a pugnar por una rnayor autonomía - ha estado abogando por una mayor coordinación entre las secretarías. Una propuesta mís radical ha sido la de crear un organismo ambiental mundial, para reunir a la gran cantidad de instituciones internacionales que actualmente influyen en la formulación de las políticas del medio ambiente, como el pNuma, el Fondo para el Medio Ambiente Munclial (FMAM), las secretarías de los diferentes acuerdos ambientales multilaterales, y muchísimos más. Lo que tha hecho el autor de la propuesta, Daniel Esty (1994.), alto funcionario del organismo de protección del medio ambiente de los Estados Unidos (EPA), no ha sido sugerir medidas prácticas para hacer recaer en una sola institución la responsabilidad de todas las negociaciones ambientales del mundo, sino que sostener que se necesita un contrapeso institucional a la omc.

Las políticas comerciales y las políticas ambientales efectivamente interactúan en formas que aún no se conocen bien y que a veces remedan un diálogo de sordos. Como lo resumió en términos muy cautelosos el Director General de la oмc, Renato Ruggiero, "es posible vislumbrar circunstancias en que el intercambio comercial, sin el apoyo de políticas ambientales bien concebidas, pudiese significar un daño para el medio ambiente -0 , por el contrario-, en que las disposiciones reglamentarias ambientales pudiesen dañar un comercio legítimo" (Ruggiero, 1995). En cada circunstancia, ¿cuál hay que ajustar? Las respuestas racionales a esta pregunta deberían surgir de investigaciones que tengan por objetivo la formulación de políticas. Al reflexionar en ese sentido, sería errado olvidar que las diferencias en los niveles de desarrollo y bienestar de los países van acompañadas por diferencias de poder de negociación en el ámbito internacional y de prioridades ambientales.

(Traducido del inglés)

\section{Bibliografia}

Adams, J. S. y T. O. McShane (1992): The Myth of Wild Africta: Conservation Without Mlusion. Nueva York, W.W. Norton \& Co.

Agosin, M. R. y D. Tussie (eds.) (1993): Trade and Growth: New Dilemmas in Trade Policy. Nueva York, St. Martin's Press.

CEPAL (Comisión Económica para América Latina y el Caribe) (1995): El Convenio de Batsilea: un excenario urgenie para Ia cooperación regional, LC/R. 1567, Santiago de Chile.

(1996): Panorama de la inserción internacional de Amé. rica Latina y el Caribe, Edición 1996, LC/G.1941, Santiago de Chile, 2 de diciembre.

CTTES (Convención sobre el Comercio Internacional de Especies Amenazadas de Fauna y Flora Silvestres) (1994): Review of alleged infractions and other problems of implementation of the Convention. Informe presentado a $I X$ Meeting of the Conference of the Parties to the Convention on International Trade in Endangered Species of Wild Fauna and Flora, Fort I-auderdale, Florida, noviembre.

Cline, W.R. (1992): The Economics of Global Warming, Washington, D.C.. Institute for International Economics (IIE).

CMMAL (Comision Mundial sobre el Medio Ambiente y el Desarrollo) (1987): Our Common Future, Oxford, Reino Unido, Oxford University Press.

Convención Marco de las Naciones Unidas sobre el Canbio Climático (1996): Activities Implemented Jointly: Annual Keview of Progress Under the Pilot Phase, FCCC/CP/1996/14, Ginebra.
Cook, E. (1996): Making a Milestome in Ozone Protection: Learning from the CFC Phase-out, Washington, D.C., Instituto de tos Recursos Mundiales, enero.

Enssley, J. (ed.) (1996): The Global Warming Debate: The Report of the European Science and Environment Forum, Dorset, Reino Unido, Bourne Press Limited.

Esty, D.C. (1994): Greening the GATT: Trade, Environment and the future, Washington D.C., IIE.

Feinsilver, J. M. (1996): Prospección de ba biodiversidad: potenciatidades para los paises en desartollo, Revista de la CEPAL, $\mathrm{N}^{\circ}$ 60, LC/G.1943-P, Santiago de Chile, CEPAL.

Klepper, G. y F. Staehler (1996): Sustainubility in Closed and Open Economies, Kiel, Alemania, The Kiel Institute of World Economics.

Low, P. (ed.) (1992): International Trade and the Environment, World Bank discussion paper, $\mathrm{N}^{0} 159$, Washington, D.C., Banco Mundial.

Markandya, A. (1994): Is free trade compatible with sustainable development?, Unc'tad Review 1994, Ginebra, Conferencia de las Naciones Unidas sobre Comercio y Desarrollo (UNCTAD). Publicación de las Naciones Unidas, No. E.94.II.D.19.

Naciones Unidas (1997): Fourth Expert Group Meeting on Finuncial Issues of Agenda 2J. Departamento de Coordinación de Política y Desarrollo Sosterible/CEPAL/Banco Interamericano y Desarrollo (BID), 8 al 10 de enero.

Opschoor, H. (1996): Sustainability: A suspicious concept, F. Duijnhouwer, M.Veldhuis (eds.), RAwOo/RMNO Lectures on 
Sustainable Development. La Haya, Paises Bajos, Advisory Council for Scientific Research in Development Problems (RAWOO).

Pearce, D. (1994): The greening of the GATT: some economic considerations, J. Cameron, P. Demaret y D. Geradin (eds.) Trade and the Environment: The Search for Balance, vol. I, Londres. Cameron May Ltd.

PNUma (Programa de las Naciones Unidas para el Medio Ambjente) Consejo de Administración (1996): International Comventions and Protocols in the Field of the Environment. Report of the Executive Director, Nineteenth session (Nairobi, 27 de enero al 7 de febrero), UNEP/GC.19/3I, Nairobi, 18 de diciembre.

PNUMA, Technical Working Group to Develop Draft Technical Guidetines on the Environmentally Sound Management of Hazardous Wastes Subject to the Basel Convention (1997a): Draft Position Paper on Hazard Characterization and Classification of Wastes Within the Framework of the Basel
Convention, Twelfth session, 24 al 28 de febrero, UNEP/CHW/ WG. $4 / 12 / 2$, Ginebra, enero.

(1997b): Considerations of Wastes Placed on List $C$. Twelfth session, 24 al 28 de febrero, UNEP/CHW/WG.4/12/4, Ginebra, 6 de enero.

Ruggiero, R. (1995): The Paul-Henri Spaak Lecture, Boston, Massachusetts, Harvard University, I6 de octubre, mimes.

Simonis, U.E. (1996): Internationally tradeable emission certificates. Linking environmental protection and development, Economics, vol. 53, Tubingen, Alemania, Institute for Scientific Co-operation.

Von Moltke, K. (1996): International Environmental Management. Trade Regimes and Sustainability, Winnipeg, Manitoba, Canadá, International Institute for Sustainable Development.

Zollinger, P. y R. C. Dower (1996): Private Financing for Globat Environmental Initiatives: Can the Climate Convention's "Soint Implementation" Pave the Way?, WRI issues and ideas, Washington, D.C., Instituto de los Recursos Mundiales, octubre. 